\title{
Effect of response effort requirement on frequency of short interresponse times
}

\author{
HARVARD L. ARMUS \\ University of Toledo, Toledo, Ohio
}

Higher levels of required response effort in an operant task resulted in a greater absolute and relative frequency of short (less than $1 \mathrm{sec}$ ) interresponse times.

Previous pilot work (Armus, 1952) presented some supporting evidence for the view that greater required response effort results in a larger ratio of short interresponse time (IRT) leverpresses to total number of presses. The present study was designed to determine whether this increasing ratio of short IRT responses to total responses was a reliable function of the response effort requirement.

\section{METHOD}

\section{Subjects and Apparatus}

The subjects were 9 experimentally naive male Long-Evans strain rats, approximately 170 days old. The apparatus was an operant chamber approximately $30 \mathrm{~cm}$ on a side, fitted with a $10-\mathrm{cm}$-wide lever mounted parallel to the front wall of the chamber and $11 \mathrm{~cm}$ above the floor. Vertical lever movement of approximately $4 \mathrm{~mm}$ was sufficient to operate the lever microswitch. The lever could be fitted with easily removable counterweights to change the response effort requirement.

\section{Procedure}

All animals were given 28 days of adaptation to a feeding schedule of $1 \mathrm{~h}$ of food every $24 \mathrm{~h}$, with ad-lib access to water. During the final

This research was partly supported by a grant from the Small Grants Program of the Graduate School of the University of Toledo.
23 days of feeding-schedule adaptation, animals were prefed $1545-\mathrm{mg}$ Noyes rat food pellets $5 \mathrm{~min}$ before the daily feeding session.

Animals were then given 10 daily 40 -min pretraining sessions in which leverpresses were reinforced on a CRF schedule with one 45-mg food pellet, with the lever-operating force set at $10 \mathrm{~g}$. Approximately 675 reinforced presses were made by each rat during the 10 days of pretraining, with approximately 100 per day on the last 4 days.

On each of the next 8 days, each rat was placed into the operant chamber for $\mathbf{4 0} \mathrm{min}$, divided into four 10-min periods, each characterized by a different response force requirement $(5,20,35$, or $50 \mathrm{~g})$.

At the end of each 10-min period, the houselight went off, and the experimenter immediately replaced the lever counterweight with the next programmed one and reset the apparatus. This procedure typically took no longer than $4 \mathrm{sec}$.

The order of response force requirements was the same for each rat on a given day, but was varied over days so that over the 8 training days, each force requirement appeared in each position an equal number of times and preceded and followed every other force requirement an equal number of times. Responses were recorded by means of an ink-writing operations recorder.

\section{RESULTS}

The effect of the effort requirement was significant, with greater required response effort resulting in fewer responses [Friedman two-way nonparametric ANOVA, $\left.\chi_{r}^{2}(3)=15.21, p<.01\right]$ but in more responses with

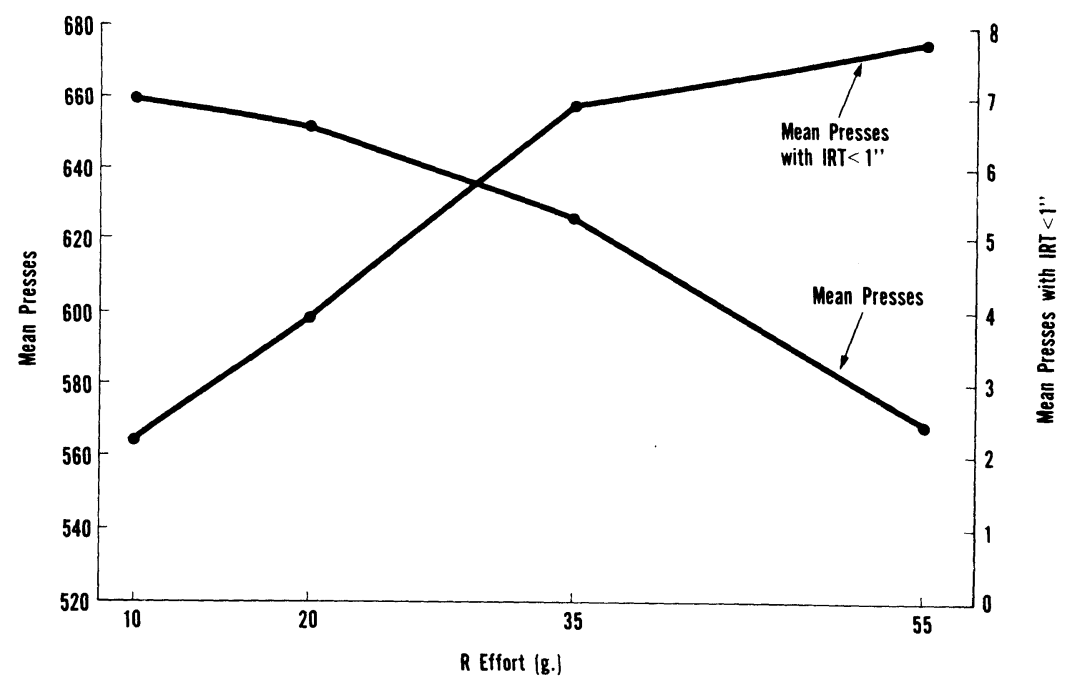

Figure 1. Mean number of presses and mean number of presses with IRT of less than 1 sec per 10-min test period as functions of minimum force (effort) required to activate response lever. 


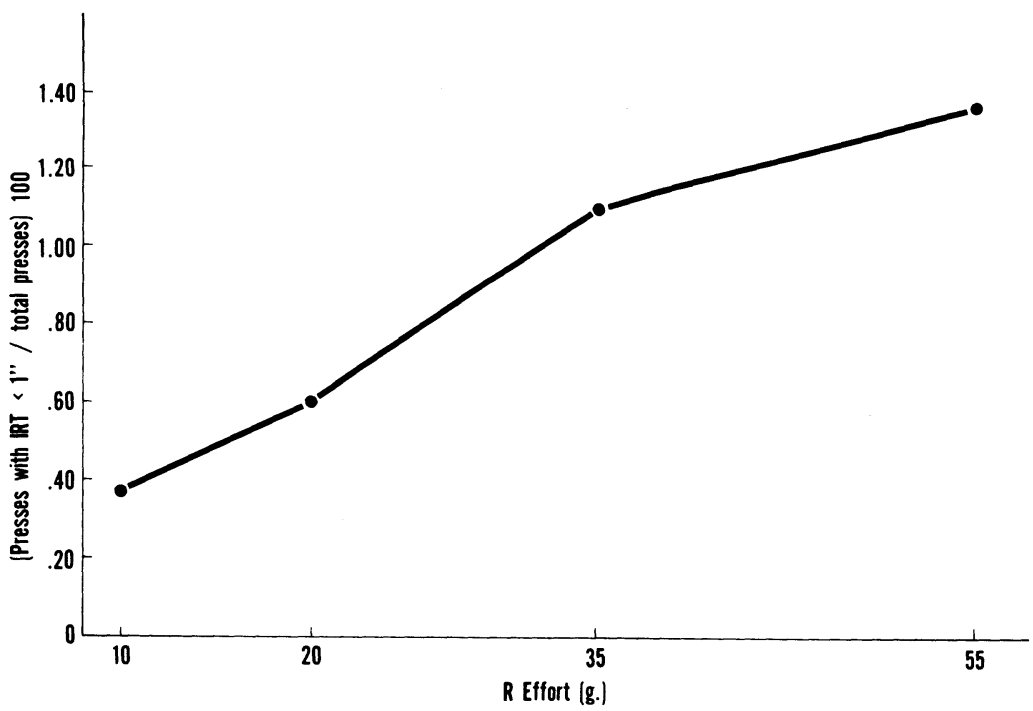

Figure 2. Ratio of presses with IRT of less than $1 \mathrm{sec}$ to total number of presses as a function of minimum force (effort) required to activate response lever.

IRTs of less than $1 \sec \left[\chi_{r}^{2}(3)=10.41, p<.02\right]$. These data are shown in Figure 1. The relation of the ratio of responses with short IRTs to total responses as a function of required response effort is presented in Figure 2. This effect is also significant $\left[\chi_{r}^{2}(3)=12.44, p<.01\right]$, with the ratio increasing as response effort increased.

\section{DISCUSSION}

The current findings are thus in line with Armus's (1952) pilot data. In an attempt to explain these findings, it is necessary to first rule out an obvious possible confounding factor, namely, differential "lever bounce" caused by the use of different counterweights. In a great many manual operations with each of the four weights, using both rapid and slow lever release, not one case of lever bounce sufficient to operate the lever microswitch was recorded, thus effectively eliminating this possible contaminant.

One plausible explanation for these findings might involve the notion of differential response-produced stimulus feedback. If stimulus feedback from a leverpress provides an important cue for the next leverpress, it is possible that more salient feedback cues might be more strongly associated with leverpressing than are less salient cues. If so, and if the feedback cues from a more effortful response are more salient than those from a less effortful response, such stronger association might lead to more short response times. If the explanation is valid, a procedure that interferes with response chaining should serve to decrease or eliminate the relationship between the response effort requirement and the occurrence of short IRTs.

It may be suggested that an explanation of these findings might be found in terms of different reinforcement schedules that may be present in the several effort conditions. It is likely that higher response effort requirements might result in a greater number of partial leverpresses (presses of insufficient lever excursion to activate the lever microswitch) and, thus, change the reinforcement schedule from continuous to partial for the higher response effort conditions. However, each complete leverpress was reinforced on a continuous schedule, and it was these complete presses that showed the increase in short IRTs with increases in required response effort. If anything, the interjection of partial leverpresses between complete ones, the situation more likely in the higher effort conditions, would lead to more long IRTs, rather than to the obtained increased frequency of short IRTs.

\section{REFERENCE}

ARMUS, H. L. (1952). Number of responses and duration of bar depression as functions of effortfulness of task. Unpublished master's thesis, Columbia University, New York.

(Manuscript received for publication March 24, 1986.) 